\title{
A novel method for screening beta-glucosidase inhibitors
}

\author{
Sony Pandey*, Ayinampudi Sree, Soumya S Dash and Dipti P Sethi
}

\begin{abstract}
Background: Few beta-glucosidase inhibitors have so far been reported from microorganisms due to the practical difficulties in performing the inhibition tests and subsequent interpretation of results. In an effort to investigate marine microbial extracts for $\beta$-glucosidase inhibitors, we developed a new protocol, using esculin as substrate in an agar plate based assay, to screen a large number of microbial extracts in a short span of time.

Results: With the new method, pale yellowish zones against the blackish brown background could be visually observed with more clarity in sample extracts where $\beta$-glucosidase inhibitor was present. The new method was compared with the closest existing method and established beyond doubt. This agar plate based procedure required about one hour for minimum 12 samples and the throughput increases with the size of the agar gel plate used.
\end{abstract}

Conclusions: The new protocol was simple, rapid and effective in detecting beta-glucosidase inhibitors in microbial extracts.

Keywords: Glucosidase inhibitor, Esculin, Conduritol $\beta$-epoxide, Microbial extracts, marine microorganisms, anti-diabetics, anti-obesity, anti-HIV

\section{Background}

Glucosidase inhibitors are responsible for disruption of the activity of glucosidase, an enzyme that cleaves the glycosidic bond. These inhibitors have played a vital role in revealing the functions of glucosidases in living system by modifying or blocking specific metabolic processes; and, this revelation led to several applications of these chemical entities in agriculture and medicine [1].

The quest for new glucosidase inhibitors is crucially important owing to their therapeutic potential in the treatment of diabetes, human immuno deficiency virus infection, metastatic cancer, lysosomal storage disease etc. [2]. Microorganisms, particularly marine microorganisms, have an unparalleled distinction of producing valuable compounds. So, screening microbial culture extracts for uncovering novel structures that can inhibit glucosidases, is of immense interest.

There are extremely few reports of glucosidase inhibitors, particularly $\beta$-glucosidase inhibitors from microorganisms, possibly because of lack of efficient high throughput methods to detect the presence of $\beta$-glucosidase inhibitors

\footnotetext{
* Correspondence: sony@immt.res.in

Environment and Sustainability Department, CSIR-Institute of Minerals and Materials Technology, Bhubaneswar 751013, India
}

in microbial culture extracts. The most commonly employed method involves $p$-nitrophenyl- $\beta$-D-glucopyranoside (PNPG) as substrate in either microplate screening test or TLC autographic method $[3,4,5]$. In this method, glucosidase activity is measured indirectly, in a colorimetric assay by visual or spectrophotometric assessment of the nitrophenyl chromophore (yellow) released from PNPG in the absence of inhibitor. The yellow colouration developed using this glucopyranoside in a glucosidase positive reaction, is too faint and not in contrast with its surrounding for clear visual distinction in TLC plate or otherwise [5-7]. Microwell plate methods are rapid, but many factors such as protease in fermentation broths, microbial contamination of extracts, biological pigments, or salts in crude extracts can interfere with the readings [8].

The TLC autographic method - using esculin as substrate by Salazar and Furlan [7] was the most convincing method as an alternative to the methods using PNPG. In this TLC autographic method, the enzyme $\beta$-glucosidase is immobilized by gel entrapment in agar and TLC autography is performed. The enzyme activity is tested on esculin (6, 7-dihydroxycoumarin 6-glucoside) as substrate which splits into esculetin (6, 7-dihydroxycoumarin) and glucose; the released esculetin reacts with $\mathrm{FeCl}_{3}$ to form a blackish 


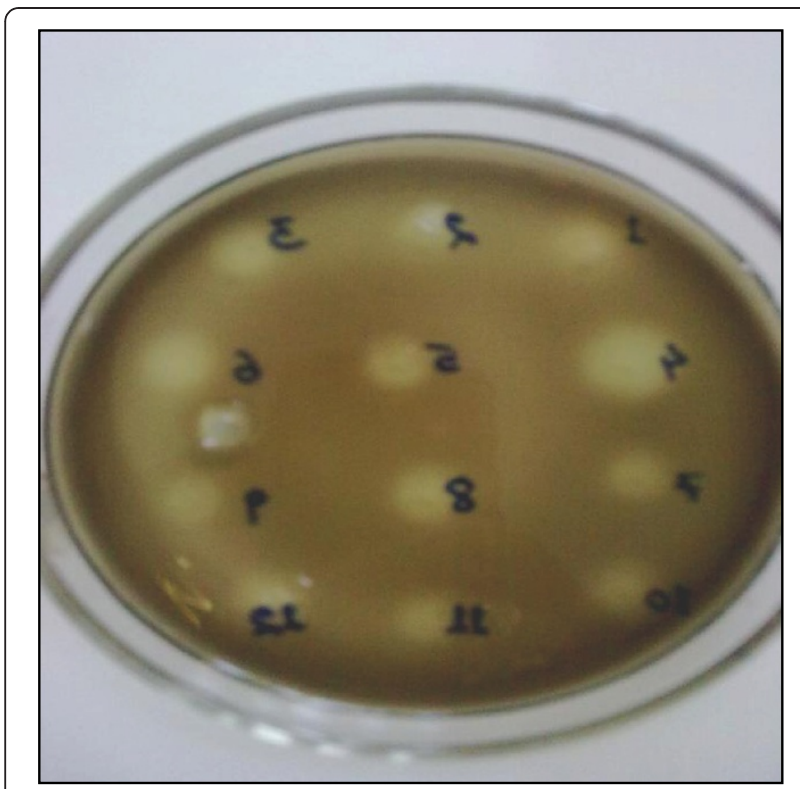

Figure $1 \beta$-glucosidase inhibition using the agar plate method developed in this study.

brown precipitate. Inhibition of this activity is observed as a pale yellowish zone around the spot of the positive samples.

Many of the previous studies have used TLC autographic method, which may not be suitable for high throughput screening as they are more laborious and time consuming. Moreover, uniform separation of compounds in all extracts cannot be achieved with single solvent system; hence spotting all the extracts on one TLC plate to rapidly perform the assay would be frustrating. For screening a large number of natural extracts,
TLC autography was performed without developing the plate so that activities resulting from synergistic action of multiple components of extracts are detected [9]. In this context, we consider the use of TLC plate to be unnecessary; more so because the zone of inhibition on white TLC plate background was not very clear and hence there are chances of losing some promising natural extracts. In a nutshell, accurate assessment of glucosidase inhibition activity in several extracts at a time is difficult by these conventional methods.

Thus, we developed a novel method by pouring the enzyme-agar solution in a thin layer on a petri dish and spot inoculating the samples on the agar surface, for achieving clear detection of $\beta$-glucosidase inhibitors in microbial culture extracts.

\section{Results and discussion}

The microbial culture extracts, which were positive for $\beta$-glucosidase inhibitors, showed as pale yellowish zone of inhibition at places where the samples were spotted while the rest of the plate turned blackish brown due to the reaction of esculetin and ferric ion (Figure 1). A large number of methanol extracts of microorganisms were screened using the new method, and we found 98 extracts (32\%) contain inhibitors out of 304 extracts tested (data not shown). As compared to the earlier reports of screening plant and microbial extracts, this method could detect greater number of positive extracts, which may be, because of the easily discernible results $[3,8]$. This method is also rapid as it takes about $1 \mathrm{hr}$ to test 12 samples in a $\varnothing 90 \mathrm{~mm}$ petri plate. The throughput can be increased by increasing petri plate size or using a multiple of plates.

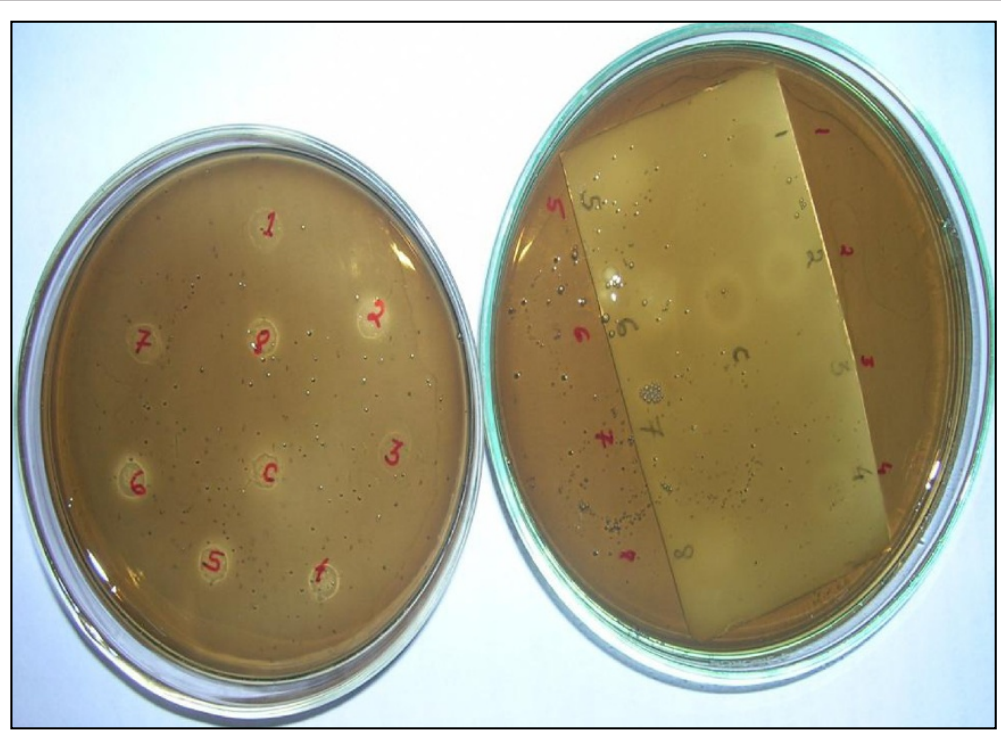

Figure 2 Side by side comparison of agar plate method with TLC autography method. Samples labelled as 1, 2, 3, 4, 5, 6, 7 and 8 are the methanol extracts of marine microorganisms and, $C$ is for control $-0.75 \mu \mathrm{g}$ conduritol $\beta$-epoxide. 
Table 1 Inhibition of $\beta$-glucosidase by different doses of conduritol $\beta$-epoxide

\begin{tabular}{cccccccc}
\hline & \multicolumn{7}{c}{ Concentration $(\mu \mathrm{g})$} \\
\cline { 2 - 8 } & 2.5 & 1 & 0.75 & 0.50 & 0.25 & 0.1 & 0.05 \\
\hline Inhibition & + & + & + & + & + & + & + \\
\hline
\end{tabular}

The present agar plate based method evolved from the protocol described by Salazar and Furlan [7], since we encountered some difficulty while screening the microbial extracts. The enzyme-agar solution did not evenly spread on the TLC plate, and the brown colour (due to esculetin reaction) on white plate background was not uniform throughout the TLC plate; thus it was difficult to observe the inhibition activity as clear spots in contrast to the surrounding. Although zones were visible, it was difficult to ascertain certain samples as positive or negative. Hence we modified the method, and used petri plates to set in the enzyme-agar solution and spot inoculated the samples on the enzyme-agar plate and dried the samples using a blow-dryer. Then the plate was flooded with substrate solution. The results were visually

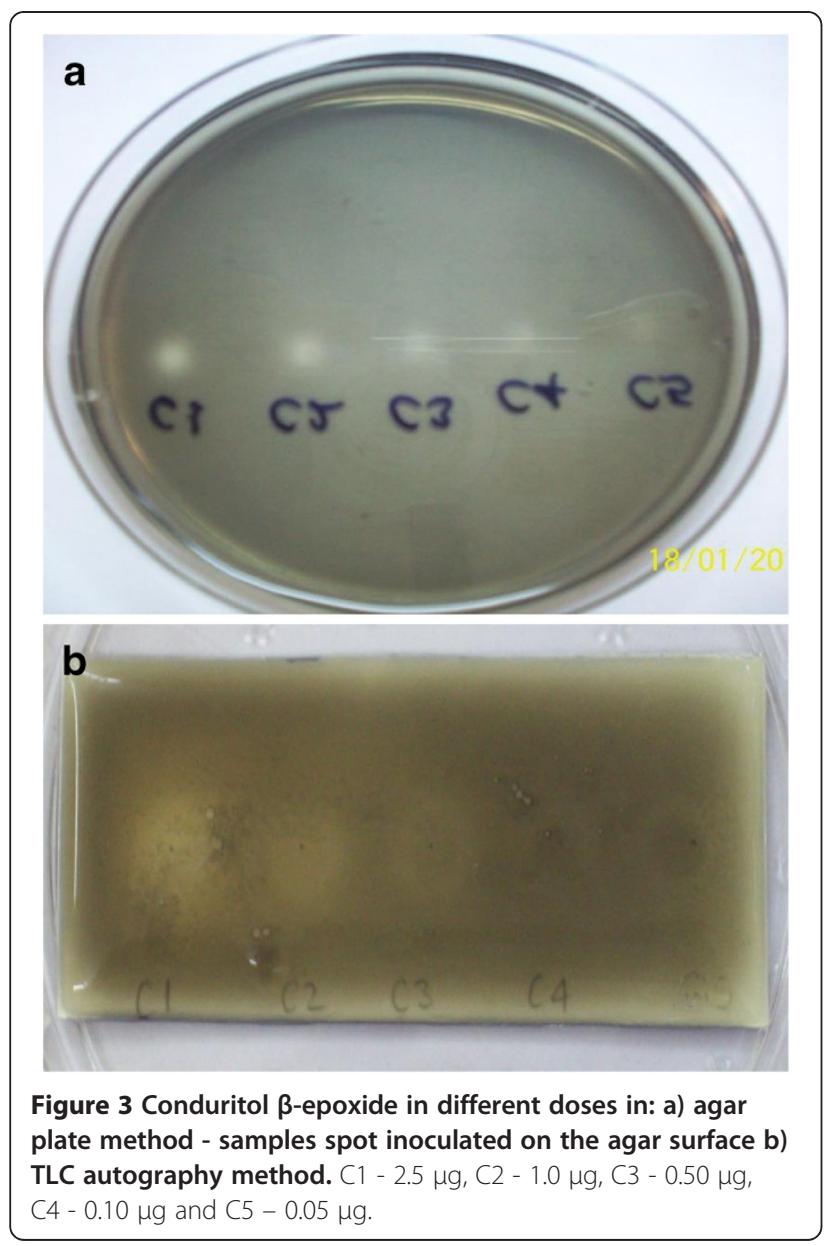

clear in this agar plate method when compared side by side with TLC autography (see Figure 2 and Figure 3).

We tested a subset of 31 samples with Salazar's method described in 2007 and 2011 [7,9], as well as with the new method. All of the 31 samples were inactive when the TLC plate was developed indicating synergistic interaction among the sample components was responsible for the positive activity. Out of the 31 extracts tested 13 were observed to be positive on the undeveloped TLC plate whereas, 16 showed $\beta$-glucosidase inhibition activity on the agar plate method. However, the quality of zone in some samples was not clear in TLC autographic method as shown in Figure 2.

Conduritol $\beta$-epoxide - an active site-directed covalent inhibitor - was tested in a dose dependent order to confirm the effectiveness of this method and the results are presented (Table 1). The minimum detection limit of conduritol $\beta$-epoxide in the new method, when samples were spot inoculated on the agar surface, is $0.05 \mu \mathrm{g}$. Although Salazar and Furlan [7] reported the detection limit to be $0.10 \mu \mathrm{g}$ in TLC autographic method, we observed similar results with conduritol in both the methods. However, the clarity of zones is undoubtedly better in the agar plate method as seen in Figure 3a and 3b.

We also tested imidazole derivatives, 1-(3-aminopropyl)imidazole and 2-aminobenzimidazole, as reversible inhibitors of $\beta$-glucosidase with this method [10]. Figure 4 demonstrates the inhibition activity of 1-(3-aminopropyl)imidazole in a dose dependent order up to $50 \mu \mathrm{g}$. The detection limit of 2-aminobenzimidazole was $100 \mu \mathrm{g}$. As compared to conduritol, imidazole derivatives are less potent inhibitors of $\beta$-glucosidase [11].

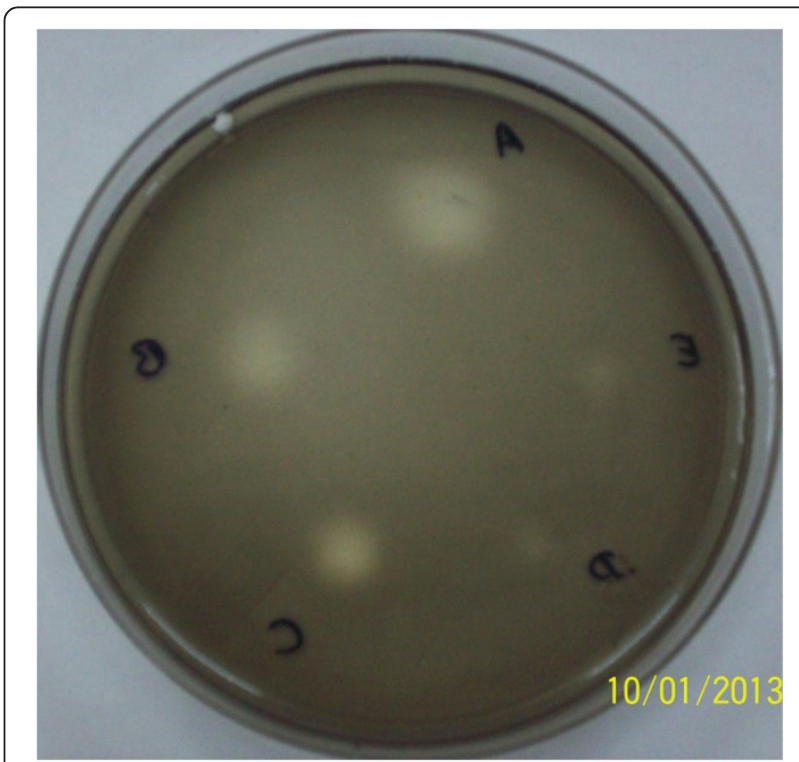

Figure 4 1-(3-aminopropyl)-imidazole in different doses. A - $2000 \mu \mathrm{g}, \mathbf{B}-1000 \mu \mathrm{g}, \mathbf{C}-500 \mu \mathrm{g}, \mathbf{D}-100 \mu \mathrm{g}$ and E- $50 \mu \mathrm{g}$. 
Comparing the new method with the protocol of Salazar and Furlan [7], we achieved reliable results in lesser time. The enzyme-inhibitor and enzyme-substrate reaction time of $2 \mathrm{hrs}$ was not necessary. The enzyme-inhibitor incubation of 15 min was sufficient as the samples were blow dried. Similarly, after pouring the esculin solution the zones could be seen within 10-15 min, which off course becomes clear as the time progresses, but within $30 \mathrm{~min}$, the contrast of zones is completely clear.

\section{Conclusions}

The new method can be used in conjunction with TLC autography. With agar plate method, several extracts could be quickly screened for activity and then the compound responsible for $\beta$-glucosidase inhibition in positive extracts could be located with the TLC autographic method. The present method is rapid and effective; hence it is suitable for initial screening. The contrast in inhibition zones is quite prominent as compared to other methods described so far for $\beta$-glucosidase inhibition. The sensitivity of this method is same or better than the TLC autographic method. It is very simple and convenient to perform.

\section{Methods}

\section{Materials}

Almond $\beta$-glucosidase enzyme $(5.2 \mathrm{U} / \mathrm{mg}$, Sigma) reconstituted in sodium acetate buffer to $2.5 \mathrm{U} / \mathrm{ml}$, $0.1 \mathrm{M}$ sodium acetate buffer ( $\mathrm{pH}-5), 0.2 \% \mathrm{w} / \mathrm{v}$ solution of esculin (HiMedia, Mumbai), $0.5 \% \mathrm{w} / \mathrm{v}$ solution of $\mathrm{FeCl}_{3}$, conduritol $\beta$-epoxide (Sigma) in $5 \mathrm{mg} / \mathrm{ml}$ solution and agar powder.

\section{Revival of cultures}

A total of 304 marine microorganisms isolated from two sponge samples and 4 sediment samples were revived from cryopreserved stocks (in 10\% glycerol) and agar slants. All the organisms grew on Nutrient Agar (HiMedia) media prepared in $50 \%$ aged natural seawater at $30^{\circ} \mathrm{C}$ within $48-72 \mathrm{hrs}$.

\section{Extraction of metabolites}

The organisms were inoculated in $50 \mathrm{ml}$ Nutrient Broth prepared in $50 \%$ natural seawater and incubated at $30^{\circ} \mathrm{C}$ in $200 \mathrm{rpm}$ shaker for $48 \mathrm{hrs}$. The culture was centrifuged (9000 rpm/20 $\mathrm{min}$ ) and the supernatant used for extraction of secondary metabolites. The supernatant collected in a $250 \mathrm{ml}$ flask was extracted by mixing $10 \%$ diaion HP20 (Sigma) and shaking for $30 \mathrm{~min}$ on a magnetic stirrer. Then the flask contents were packed in a glass column and washed with $15 \mathrm{ml}$ distilled water. Finally, the metabolites on diaion were eluted with $20 \mathrm{ml}$ methanol. The collected methanol fractions were evaporated in a rotary evaporator (Heidolph, Germany), dissolved in DMSO and stored at $-20^{\circ} \mathrm{C}$.

\section{Inhibition assay}

An enzyme agar solution containing $\beta$-glucosidase was prepared in $7 \mathrm{ml}$ sodium acetate buffer with $0.07 \mathrm{~g}$ of agar powder dissolved at $80-100^{\circ} \mathrm{C}$; followed by the addition of $1.2 \mathrm{ml}$ of $\mathrm{FeCl}_{3}$ solution and $40 \mu \mathrm{l}$ of enzyme $\beta$-glucosidase at $60^{\circ} \mathrm{C}(0.01 \mathrm{U} / \mathrm{ml})$. The final volume was adjusted to $10 \mathrm{ml}$ with the acetate buffer. An aliquot of 8-10 ml solution was poured into petri plates and allowed to set. The samples - $5 \mu$ l of the extract - were spot inoculated with a micropipette on the surface of the agar plate and blow dried or air dried. Alternately, the samples can be loaded on sterile filter paper discs, dried and placed on the agar plate. The plates were incubated at room temperature for $15 \mathrm{~min}$ for primary reaction between the enzyme and inhibitor. Later on, 6-7 $\mathrm{ml}$ of esculin solution was added to cover the surface of agar and again incubated at room temperature for $30 \mathrm{~min}$ for enzyme-substrate reaction. In case, paper discs are used they have to be removed before adding esculin. Conduritol $\beta$-epoxide, an irreversible inhibitor, in concentrations 2.5 , $1,0.75,0.50,0.25,0.10$ and $0.05 \mu \mathrm{g}$ was used as a positive control and DMSO without extract as negative control. 1-(3-aminopropyl)-imidazole and 2-aminobenzimidazole were used as reversible inhibitor control, in concentrations 2000, 1000, 500, 100 and $50 \mu$ g. Clear zones of inhibition were recorded by measuring the zone size. A subset of 31 samples was also compared using this agar plate method and TLC autographic method with or without developing the TLC plate. These experiments were repeated thrice with some extracts to check the reproducibility of the method.

\section{Competing interests}

The authors declare no competing interests.

\section{Authors' contributions}

SP contributed to the design of experiments, acquisition, analysis and interpretation of data, and drafting the manuscript. AS contributed in the conception of work on beta-glucosidases, sample collection and editing of the manuscript. SSD and DPS helped in execution of experimental work and acquisition of data. All authors have read and approved the final manuscript.

\section{Acknowledgements}

The authors are thankful to Council of Scientific and Industrial Research (CSIR, India) for funding the work and Director CSIR-IMMT for the infrastructure support. We gratefully acknowledge Dr. Tapan Chakrabarti, former Head and founder of Microbial Type Culture Collection - an International Depository Authority, Chandigarh, India, for critically examining the manuscript. We heartily thank Dr. B.P. Bag, Senior Scientist, CSIR-IMMT, Bhubaneswar, for providing us the imidazole derivatives used in the experiments.

Received: 26 June 2012 Accepted: 1 March 2013

Published: 8 March 2013

\section{References}

1. Asano N: Glycosidase inhibitors: updates and perspectives on practical use. Glycobiology 2003, 13:93R-104R.

2. de Melo EB, Gomes AS, Carvalho I: $\alpha$ - and $\beta$-Glucosidase inhibitors: chemical structure and biological activity. Tetrahedron 2006, 62:10277-10302. 
3. Atsumi $\mathrm{S}$, Umezawa $\mathrm{K}$, linuma $\mathrm{H}$, Naganawa $\mathrm{H}$, litaka $\mathrm{Y}$, Takeuchi $\mathrm{T}$ : Production, isolation and structure determination of a novel $\beta$ glucosiadse inhibitor cyclophellitol, from Phellinus sp. J Antibiot 1990, 43:49-53.

4. Paramitha VS, Lipton AP, Thangaraj M: Evaluation of $\alpha$ - and $\beta$ - glucosidase inhibitory properties of macro-algae using intestinal extracts of marine snail, Thais rudolphi (Lamarck, 1822). Indian J Biotechnol 2008, 7:61-65.

5. Simões-Pires CA, Hmicha B, Marston A, Hostettmann K: A TLC bioautographic method for the detection of $\alpha$ - and $\beta$-glucosidase inhibitors in plant extracts. Phytochem Anal 2009, 20:511-515.

6. Kwon KS, Lee J, Kang HG, Hah YC: Detection of $\beta$-glucosidase activity in polyacrylamide gels with esculin as substrate. Appl Environ Microbiol 1994, 60:4584-4586.

7. Salazar MO, Furlan RLE: A rapid TLC autographic method for the detection of glucosidase inhibitors. Phytochem Annal 2007, 18:209-212.

8. Chen $H$, Yan $X$, Lin W, Zheng L, Zhang W: A new method for screening aglucosidase inhibitors and applications to marine microorganisms. Pharm Biol 2004, 42:416-421.

9. Salazar MO, Micheloni O, Escalante AM, Furlan RLE: Discovery of a $\beta$ glucosidase inhibitor from a chemically engineered extract prepared through sulfonylation. Mol Divers 2011, 15:713-719.

10. Li YK, Byers LD: Inhibition of beta-glucosidase by imidazoles. Biochim Biophys Acta 1989, 999(3):227-232.

11. Field RA, Haines AH, Chrystal EJT, Luszniak MC: Histidines, histamines and imidazoles as glycosidase inhibitors. Biochem J 1991, 274:885-889.

doi:10.1186/1471-2180-13-55

Cite this article as: Pandey et al:: A novel method for screening betaglucosidase inhibitors. BMC Microbiology 2013 13:55.

\section{Submit your next manuscript to BioMed Central and take full advantage of:}

- Convenient online submission

- Thorough peer review

- No space constraints or color figure charges

- Immediate publication on acceptance

- Inclusion in PubMed, CAS, Scopus and Google Scholar

- Research which is freely available for redistribution 